\title{
TOWARDS REDUCING THE IMPACT OF LOCALISATION ERRORS ON THE BEHAVIOUR OF A SWARM OF AUTONOMOUS UNDERWATER VEHICLES
}

\author{
Tarek El-Mihoub, Christoph Tholen, Lars Nolle ${ }^{\bowtie}$ \\ Jade University of Applied Sciences, Department of Engineering Science, Germany \\ \{tarek.el-mihoub, christoph.tholen, lars.nolle\}@jade-hs.de
}

\begin{abstract}
Localisation errors have a great impact on Autonomous Underwater Vehicles (AUVs) as search agents. Different approaches for solving the localisation problem can be used and combined together for greater accuracy in estimating AUVs' locations. The effect of localisation errors on locating a target can be lightened by designing a search algorithm that avoids extensive use of exact location information. In this paper, two cooperative search algorithms are proposed and evaluated. In these algorithms, a high-level mechanism is employed for building a global view of the search space using minimum possible search information. These algorithms rely on low-level search algorithms with exploring roles. Particle Swarm Optimisation (PSO) and all-to-one Self-Organising Migrating Algorithm (SOMA) are selected as high-level mechanisms. The conducted experiments demonstrate that both algorithms show a robust behaviour within a range of localisation errors.
\end{abstract}

Keywords: Localisation errors, submarine groundwater discharge, search algorithms, particle swarm optimisation, self-organising migrating algorithm.

Received: 28 January 2020 Accepted: 17 June 2020 Published: 21 December 2020

\section{Introduction}

A small swarm of Autonomous Underwater Vehicles (AUVs) can be used for developing a flexible and low cost environmental marine observatory [14]. AUVs equipped with suitable sensors for collecting environmental data can explore intermediate size areas to locate phenomena of interest, like hydrothermal vents, hazardous substances and organisms [20]. AUVs have the capabilities to investigate previously inaccessible marine environments. However, due to their limited energy capacity, AUVs have a limited search range. This range can be extended to enable locating a search target by collaboration between a swarm of AUVs, which act as an environmental marine observatory. Such an environmental marine observatory can be used, for example, to locate submarine groundwater discharges (SGDs) in coastal waters. These discharges consist of in-flow of fresh groundwater mixed with seawater recirculation [8]. The SGDs discharge continuously nutrients into the coastal environment. Colour, temperature, salinity and the concentration of some chemical elements can be used as indirect indicators to track the dispersal of SGDs and to locate them [9].

\section{Localisation Problem}

Autonomous mobile robots use their current location to navigate in their mission space. A robot estimates its own location and the uncertainty of the estimation through a localisation process. Localisation has also a critical role in collision avoidance, motion planning, data collecting, map building and searching. A robot can estimate its location by sensing the environment or/and monitoring its own motion. The estimation errors depend on the details of the localisation task, the environment, the signals used for sensing the environment, the motion and the environment sensors. A map of the mission environment can simplify the localisation process and reduce the estimation errors. Without a map, the localisation process becomes a complex process as estimating land-mark locations becomes a part of it.

For AUVs, localisation is particularly essential for their recovery. It is also challenging as AUVs operate autonomously in a highly unstructured environment. The attenuation in the signals of the Global Positioning System (GPS), bandwidth limitation and communication problems aggravate the localisation problem.

AUVs use different approaches to solve the localisation problem, for example by utilising the environment as a position reference [13]. Inertial Navigation Systems (INS) [2] and Doppler Velocity Logs (DVLs) systems [18] accumulate motion estimation to determine the current location with reference to the starting location. These techniques are prone to integration drift with time [11]. Resurfacing to receive a GPS signal is used to reset the drift. However, resurfacing is not always possible, for example when operating under pack ice. External references can alleviate degrading the accuracy of the location with time.

Acoustic systems are used to estimate the absolute location of an AUV. Short baseline (SBL), ultra short baseline (USBL) and long baseline (LBL) acoustic systems are not prone to error accumulation inherent in 
motion estimation approaches [13]. In LBL systems, widely separated acoustic transponders are placed at known locations under the sea surface. Whereas USBL and SBL systems localise an AUV relative to a support vessel. The support vessel is equipped with three or more GPS-calibrated transponders in the case of SBL systems and one GPS-calibrated transponder for USBL systems. The main disadvantages of these systems is the need for external acoustic sources. In addition, the range of the acoustic systems is limited and depends on the acoustic characteristics of the environment [13].

All the above methods are error prone. Different techniques can process the location data produced by different localisation methods for more accurate localisation. Kalman filters [5], particle filters [4] and simultaneous localisation and mapping (SLAM) [6] are examples of these techniques. The fusion of location information can improve robustness, resolution and reduce localisation errors, but cannot eliminate them [12].

\section{Potential Cooperative Search Algo- rithms}

A search strategy that facilitates collaboration between a swarm of AUVs is needed to maximise the possibilities of locating a target [10], [7]. A cooperative search strategy should take into consideration the characteristics of the environment, the agents and the target [3]. Particle Swarm Optimisation (PSO) has been used as a collaborative search strategy [16]. However, it might face a premature convergence problem and can be distracted by localisation and the communication errors [16].

A single AUV in the presence of localisation errors can successfully locate a search target using search strategies that are based on coverage path planning [3]. These algorithms are also robust against premature convergence in a multiple SGDs environment. These algorithms, which use location information less heavily in deciding next search actions, have a higher probability of success.

This paper investigates the possibilities of extending the concept of alleviating the localisation problem used in [3] to cooperative search strategies. Based on this concept, the best potential cooperative strategies are those, which produce new solutions using the smallest possible set of solutions. For example, PSO and allto-one SOMA [19] can be good candidates to ease the localisation problem.

PSO models the behaviour of social entities for collaboration to locate a target [15]. Each particle moves towards the target by amending its velocity. The particle's velocity depends on its current and the best position, and the best-known position of the swarm.

$$
\vec{v}_{i+1}=\vec{v}_{i} \cdot \bar{\omega}+r_{1} \cdot c_{1}\left(\vec{p}_{\text {best }}-\vec{p}_{i}\right)+r_{2} \cdot c_{2}\left(\vec{g}_{b e s t}-\vec{p}_{i}\right)
$$

Where:

$\vec{v}_{i+1}$ and $\vec{v}_{i}$ : new and current velocity of a particle

$\bar{\omega}:$ inertia weight

$\vec{c}_{1}$ and $\vec{c}_{2}$ : cognitive and social scaling factors

$\vec{r}_{1}$ and $\vec{r}_{2}$ : two random numbers from range $[0,1]$

$\vec{p}_{i}$ and $\vec{p}_{\text {best }}$ : current and best known position of a particle

$\bar{g}_{\text {best }}$ : best known position of the swarm

The new position of a particle $\vec{p}_{i}$ be calculated as follows:

$$
\vec{p}_{i+1}=\vec{p}_{i}+\vec{v}_{i+1} \cdot \Delta t
$$

Where:

$\vec{p}_{i+1}$ and $\vec{p}_{i}$ : new and current position of a particle $\Delta t:$ time step

Self-Organising Migrating Algorithm (SOMA) is a population-based algorithm, which mimics the social behaviour of a group of entities [19]. The entities in all-to-one SOMA move towards the leader (the entity with the best fitness) while searching. The new positions of an entity, i.e. the potential new solutions, depend on its current position and the position of the leader.

$$
\begin{gathered}
X_{i, j, t}^{M L+1}=X_{i, j, s t a r t}^{M L}+\left(X_{i, j}^{M L}-X_{i, j, s t a r t}^{M L}\right) t \cdot \text { PRTVector }_{j} \\
\operatorname{PRTVector~}_{j}(n)= \begin{cases}1, & \text { if } r n d(n)>p r t \\
0, & \text { if } r n d(n)<p r t\end{cases}
\end{gathered}
$$

Where:

$M L: \quad$ current migration round

$X_{i, j, s t a r t}^{M L}$ : position of an individual at the beginning of current migration

$X_{i, j}^{M L}: \quad$ position of the leader

$t$ : jumping step, which defines the granularity of movement

PRTVector $_{j}$ : perturbation vector - its elements are randomly assigned to 0 or 1

prt : perturbation probability

Based on their use of search information, the performance of all-to-one and PSO is expected to be less sensitive to localisation errors compared with other population-based algorithms.

\section{Outlines of a Cooperative Search algo- rithm}

A cooperative search strategy that is robust against localisation errors can improve the accuracy of locating a target by a swarm of AUVs. Based on the assumption that minimising the use of location information can reduce the influence of localisation errors 
on the search, the selection for potential algorithms is narrowed to PSO and all-to-one SOMA. Both algorithms need less search information than other algorithms. The algorithms use the exact location of a solution and the relative location of one or two solutions. This also means a minimum need for communication between search agents and both algorithms are suitable for AUVs where communication problems are expected.

Developing a reliable cooperative search of a swarm of AUVs necessitates considering constrains imposed by using AUVs as search agents [3]. The limitation in the number of AUVs, the energy and the limitation on movement can lead to modifying the potential search strategy. The rest of this section shed some light on aspects that have been considered in developing the proposed cooperative strategies.

\subsection{Exploration with a Small Swarm}

Fair representation of different search regions is essential for the success of any population-based search algorithm. The population size should be large enough to maintain diversity to escape local optima. However, in the current project, the number of search agents is limited. Only three AUVs can be used as search agents. For both PSO and all-to-one SOMA, the possibilities of failure is high with only three search agents [15]. A possible way to overcome this problem is to make AUVs focus on exploration more than exploitation. PSO or SOMA as a high-level mechanism can capture a global view of the search space utilising the agents' search information.

Agents can adopt search strategies, which are based on coverage path planning [3], as low-level search algorithms. Preplanned search paths can be assigned to agents to cover the search space. A search agent follows its search path and collects information with the aim of locating the target. The agent can adhere to its predefined path without utilising local information to amend its path as the case in the Pure Preplanned Path (PPP) algorithm (algorithm-C in [3]). The agent can also exploit its own information to amend its path, slightly, for further exploration of the promising regions as in the Adaptive Preplanned Path (APP) algorithm (algorithm-A in [3]).

Samples of solutions collected by the agents can be utilised by a high-level mechanism to build a good global view. The high-level mechanism processes these samples to produce better information and share it with search agents. A search agent can amend its search path based on the global search information using its own technique. Following its own path enables an agent to complete its search mission even in the case of no gradient information or in the case of communication problems. Figure 1 shows pseudocode of the proposed framework for both algorithms.

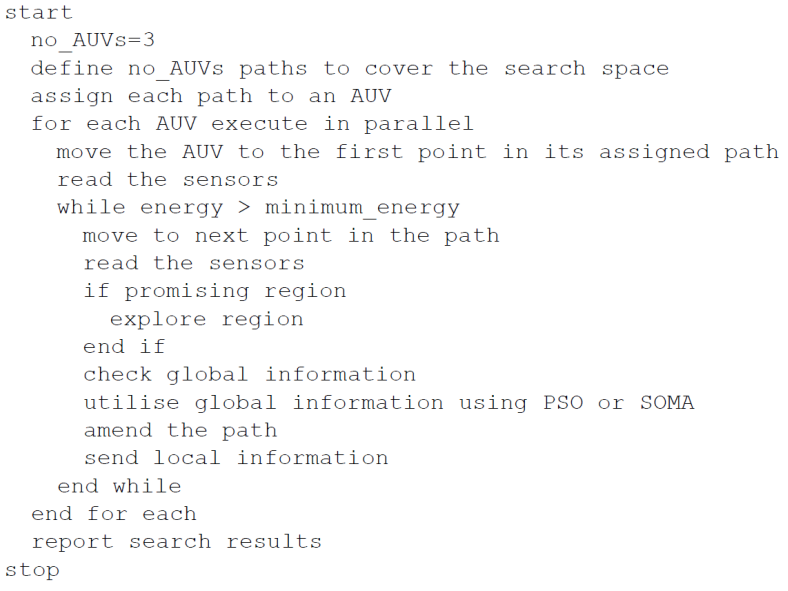

Figure 1: Pseudocode of the proposed framework for robust cooperative swarm.

\subsection{Decision for Exploration or Exploitation and Energy}

A wise use of the AUV's energy is required to ensure exploring the whole search space. However, because of the small number of AUVs and because of the exploitation capabilities of PSO and SOMA, the energy of the AUV might be completely consumed while visiting only a small region of the search space. For appropriate utilisation of the energy of an AUV, the decision to use the global information can be left to the AUV. The AUV can decide on amending its path based on the available energy and the value of the global information. By acquiring the global search information, the AUV has all necessary information for taking a good decision.

In both algorithms, two AUVs move in the area around the best performing AUV, aiming to take its place. Such a competition between the three AUVs, can lead to wasting the energy resources in a small area of the search space. It might be better from energy consumption perspective to restrict such competition between the AUV with the best solution and the AUV with the worst solution. It is also possible for each AUV to utilise global information, probabilistically, to avoid becoming trapped in a small area. The AUV can decide to utilise global search information and amend its path depending on the available energy, the value of global information, the rank of its best-found solution and the probability of utilising global information.

Validating the cooperative search decision requires moving the AUV to the suggested location. Immediate validation necessitates moving to that location. The energy consumed depends on the distance between the current location and the suggested location. The AUV can delay the validation by moving to the suggested location from the nearest location in the preplanned search path. This delay can save the AUV's energy. However, it can delay utilising new search information. The preliminary results, not shown in this paper, demonstrate that such a delay can degrade the performance of the cooperative algorithms. 


\section{Simulations}

Simulations were used to evaluate the performance of the proposed cooperative algorithms and to determine the impact of the localisation problem on their performance.

\subsection{Test Environment}

The problem of locating the SGD with the highest discharge rate in a marine environment with the dimensions of $400 \mathrm{~m} \times 400 \mathrm{~m}$ is simulated by a two-dimensional search space. In this space, an SGD is represented by a Gaussian shape with maximum temperature at its centre. The temperature of the water outside SGDs equals to the average water temperature (i.e. no gradient information). A single SGD or two SDGs with different discharge rates are located randomly in the search space. The average temperature was set to $30^{\circ}$ with a Gaussian noise of standard deviation of $0.1^{\circ}$. The maximum difference between the average temperature and the temperature of the centres of the SGDs were set to $6^{\circ} \mathrm{C}$ and $4^{\circ} \mathrm{C}[1]$. The radiuses of the basin of SGDs were selected randomly in the range from 10 to $20 \mathrm{~m}$. These radiuses are smaller than the maximum distance between the path of preplanned algorithm and the SGD [14].

\section{Simulation of AUVs as Search Agents}

Three AUVs are assigned the task of locating the centre of the SGD, which has the highest discharge rate. Each AUV uses a temperature sensor with an accuracy of $0.01^{\circ} \mathrm{C}$ to trace the SGD's location. Each AUV has a power supply that is sufficient for travelling a distance of 4500 meter with an average speed of $1 \mathrm{~m} / \mathrm{s}$ [14]. Changing the direction of the AUV costs energy and a change by an angle of $180^{\circ}$ is assumed to be equivalent to travelling 4 meters [3]. The localisation error is modelled using a Gaussian probability distribution with a standard deviation of $0,0.1,0.2$ and 0.3 meter. Every second, the AUV senses the water temperature and estimates its location. The current estimated location is used to navigate to the next location of the search path.

In the experiments, an AUV follows its assigned search path. Each AUV works in parallel with other AUVs. The parallel computing toolbox of MATLAB was used to implement the simulation. Each AUV sends the highest temperature together with the location of that temperature to other AUVs.

For the PSO-based cooperative search algorithm, the cognitive scaling, the social scaling and the inertia weight parameters were set to $0.5,1.5$ and 1.5 , respectively [15]. The worst performing AUV uses global information to generate a new solution with a probability of 0.1 . The location of new solution is the next destination of the AUV. The AUVs travel with their maximum velocity (i.e. $1 \mathrm{~m} / \mathrm{s}$ ).
For the SOMA-based cooperative strategy, the values of the jumping step, the perturbation probability and the path length parameters were set $0.3,0.5$ and 1.9 , respectively [17]. With these values of the jumping step and the path length, six new solutions are generated by the high-level mechanism at each iteration. The worst performing AUV uses the global information to generate new solutions with a probability of 0.1. The locations of steps in the direction of the leader are inserted next to the current location in the search path of the AUV.

\subsection{Performance Criterion}

The accuracy in locating the global SGD is used as the performance criterion in the simulations. The global SGD is the SGD, whose centre has the highest temperature. The accuracy is measured as the distance between the exact location of the global SGD's centre and the reported location by the algorithm. The standard deviation in achieving this accuracy for different localisation error distributions is used to show the sensitivity of the algorithms to localisation errors.

In these simulations, the performance of the cooperative algorithms were evaluated and compared with a non-cooperative algorithm. In the latter case, the AUVs follow their predefined search paths without sharing any global information.

\section{Results and Discussion}

Different sets of experiments have been conducted to evaluate the robustness of the cooperative behaviour of the proposed algorithms. In these experiments, the performances of the algorithms are compared using two different low-level search algorithms. The first algorithm is the Pure Preplanned Path (PPP) algorithm. The second algorithm is the Adaptive Preplanned Path (APP) algorithm.

The performance was evaluated in the presence of localisation errors of Gaussian nature with different standard deviation values. Each experiment was repeated for 100 times with each localisation error distribution. The results of these experiments were used to extract the cumulative distribution of the accuracy of each algorithm. The performance of each algorithm is visualised as the cumulative distribution of the best-found accuracy with its standard deviation for different localisation error distributions. In this section, the results of the conducted experiments are summarised and discussed.

\subsection{Cooperative PPP Agents}

The first set of experiments focus on the exploitation capabilities of the cooperative search algorithm employed. In these experiments, the PPP algorithm is used as a low-level search algorithm. The performances were compared on fitness landscapes with one and two SGDs. 


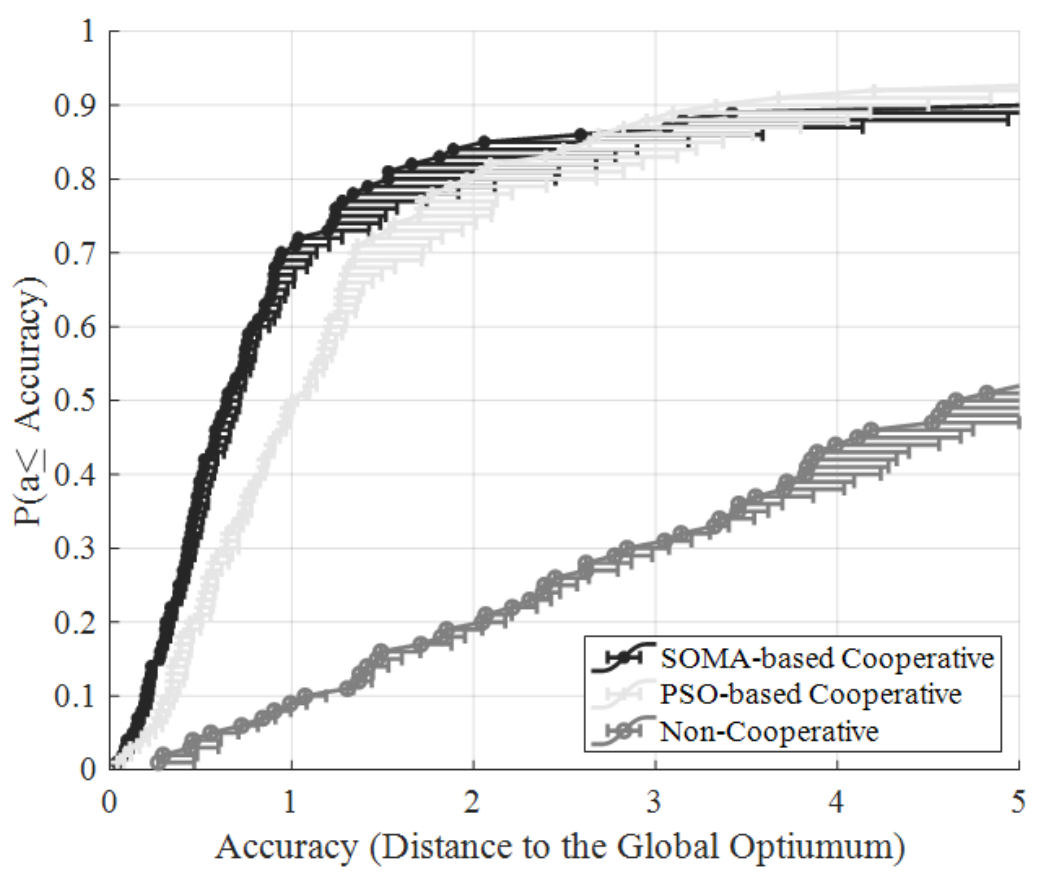

Figure 2: The performance on a single SGD environment using the PPP algorithm.

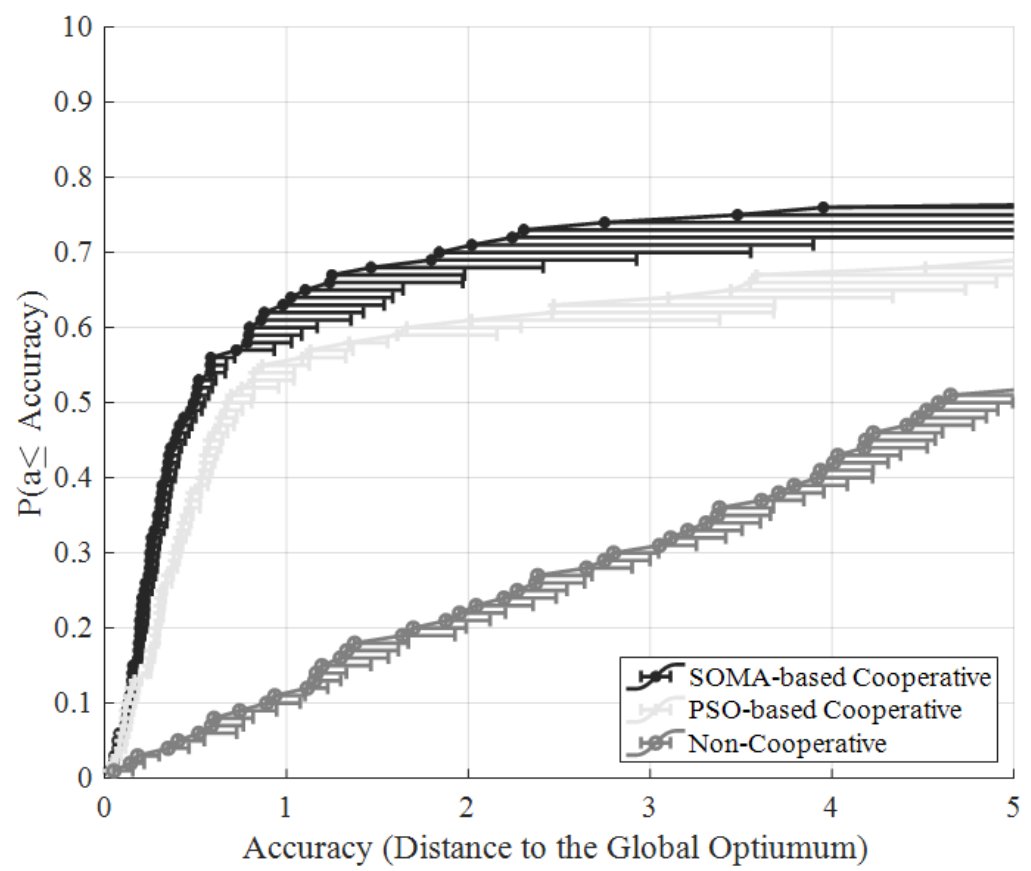

Figure 3: The performance on a two SGDs environment using the PPP algorithm.

Figure 2 shows the performance of the algorithms on a search space with a single SGD. The probabilities of locating the global optimum with different accuracies for the algorithms are depicted in the figure. The standard deviation in the achieved accuracy for each probability is also shown in the graph as horizontal line. The graphs show that both cooperative algorithms have higher probabilities of locating the global optimum with higher accuracy compared with the non- cooperative algorithm. The figure also shows that the SOMA-based algorithm slightly outperforms the PSObased algorithm. The graph shows that the standard deviations in the accuracy of the non-cooperative algorithm is less than that of the cooperative algorithms. In other words, the non-cooperative algorithm is less sensitive to localisation errors than the cooperative ones. However, the cooperative behaviour of both algorithms is robust against localisation errors, as they 


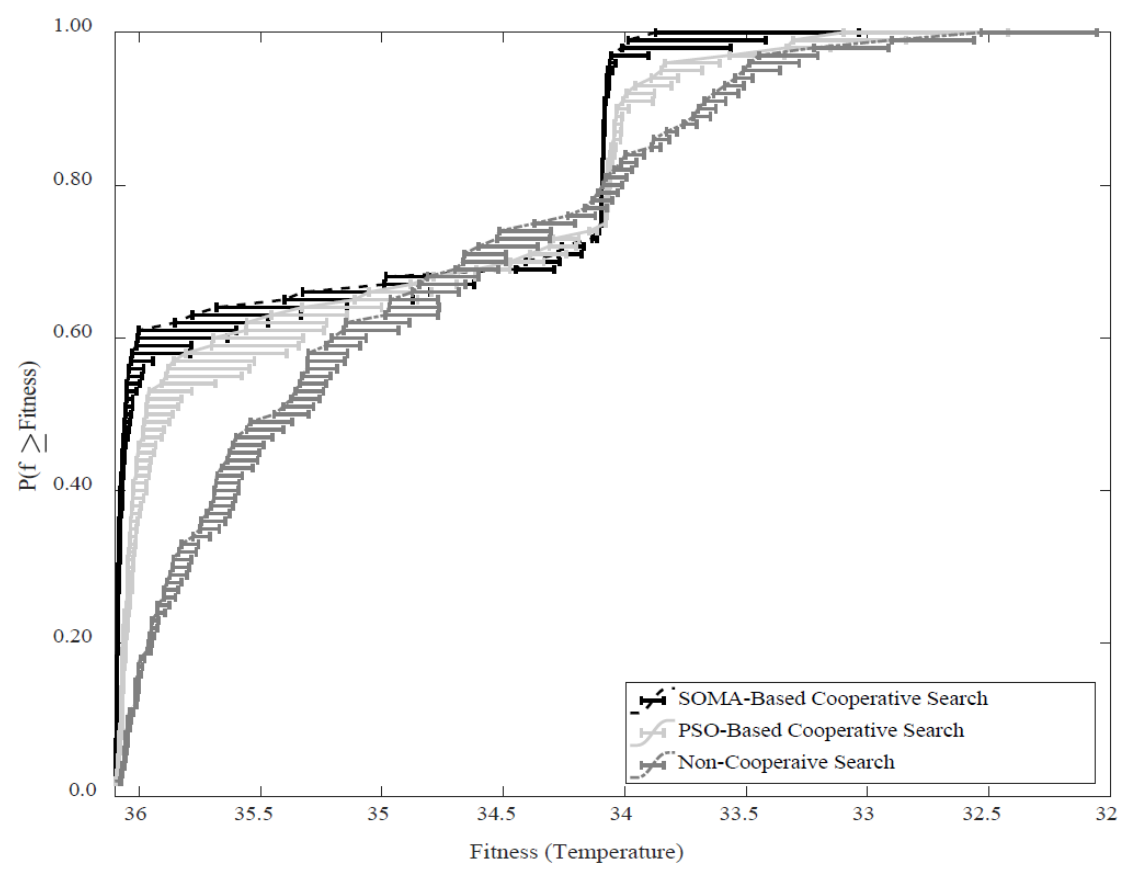

Figure 4: The performance on a two SGDs environment in terms of fitness.

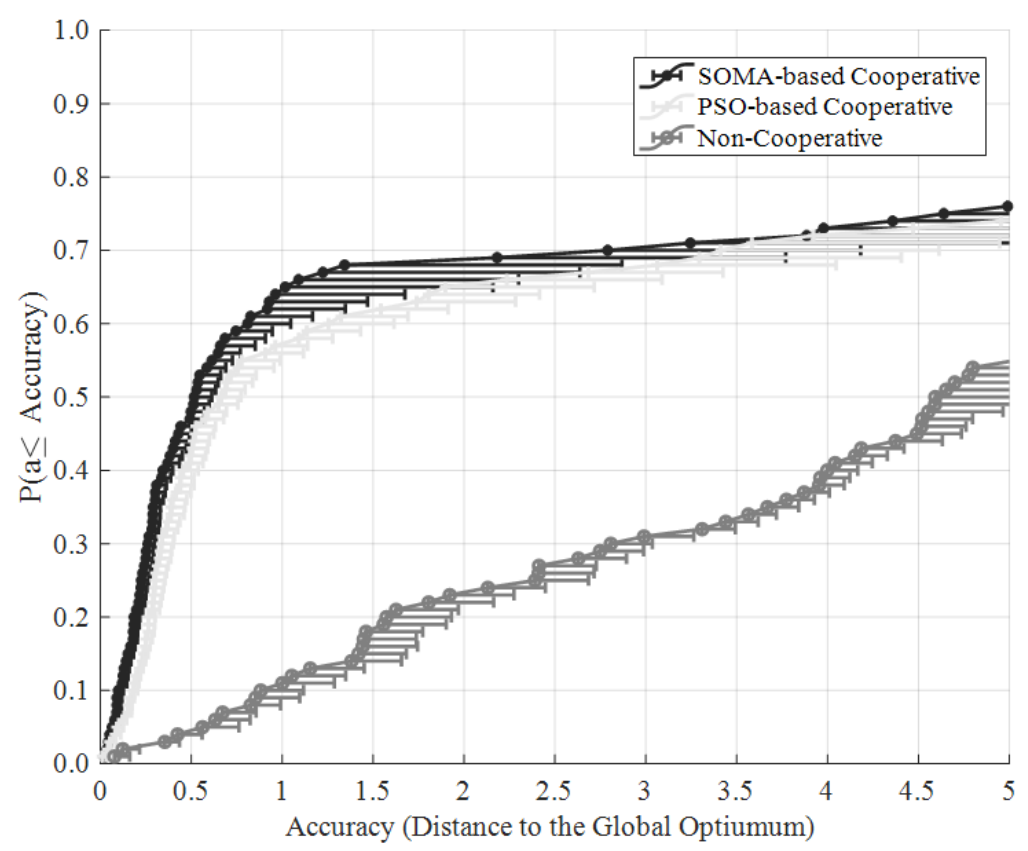

Figure 5: The performance on a two SGDs environment using the APP algorithm.

still outperform the non-cooperative one. The figure also shows that the SOMA-based algorithm is less sensitive to localisation errors compared with the PSObased algorithm in terms of their ability to locate the global optimum.

Figure 3 compares the performance of the algorithms on a search space with two SGDs. It also shows the deviations in the accuracy of locating the global optimum by different algorithms for different localisation errors. The graphs show that the SOMA-based cooperative algorithm has the best performance in more than
$60 \%$ of the experiments even with localisation errors of a standard deviation of up to 0.3 meter. It also illustrates that the probabilities of finding the global optimum with an accuracy of less than 1 meter by the SOMA-based algorithm is more than 0.55 , and more than 0.5 by the PSO-based algorithm compared with only 0.51 for the non-cooperative algorithm. The figure also shows that for accuracies of more than 5 meters, the difference in the probabities between the cooperative and the non-cooperative algorithms reduces to 0.15 . 
Figure 4 shows the highest reported fitness (temperature) for the different algorithms. The error bars in the figure show the deviation in the fitness for different localisation errors. The exploitation capabilities of the SOMA- and PSO-based cooperative algorithms are demonstrated in this figure. Once an AUV passes by an SGD, the cooperative algorithm can locate its centre with high accuracy. The exploitation ability of the SOMA-based algorithm can explain its superiority over the PSO-based in locating SGDs with higher accuracy. The SOMA-based algorithm generates a set of points in the search space between the current location and the location of the best solution. These points lie in different directions with respect to the current solution. On the other hand, the PSO-based algorithm generates a single point towards the location of the best solution and the AUV senses the temperature on the path to this new generated location.

\subsection{Cooperative APP Agents}

The experiments conducted using the PPP algorithm demonstrate the ability of the proposed high-level mechanisms to utilise global information to locate the global optimum. The APP algorithm outperforms the PPP algorithm in the presence of localisation errors [3]. Incorporating the APP algorithm can further improve the collaborative behaviour of the swarm against the localisation problem. To validate this assumption, the proposed SOMA and PSO based algorithms are combined with the APP algorithm. The benefits of such combination is evaluated by testing its ability to locate the global optimum in a search space with two SGDs.

Figure 5 shows the performance of the cooperative and the non-cooperative algorithms when combined with the APP algorithm. The graphs show that introducing the APP algorithm improves slightly the performance of the three algorithms. However, the improvement in the PSO-based cooperative algorithm is the most among these algorithms. Introducing the APP algorithm into the PSO-based algorithm increases the probability of locating the global SGD. It reduces the gap in the performance between the PSO-based and SOMA-based algorithms.

\section{Conclusion and Future Work}

The performance of the proposed cooperative algorithms demonstrates that the effect of the localisation problem can be alleviated by light use of location information. The superiority of SOMA over the PSO-based algorithm can be explained by their use of location information and their exploitation abilities. The SOMAbased algorithm needs the current location of the AUV together to the best-found location by the swarm to move towards a better location. In addition to the information that is needed by the SOMA-algorithm, the PSO-based algorithm needs the best-found location by the AUV. The PSO-based algorithm also implicitly uses the accumulated location information to determine the particle current speed. Furthermore, the SOMA-based algorithm explores the space around the best solution and the PSO-based algorithm explores only the path from the current solution towards the best solution.

Incorporating the APP algorithm improves the ability of the low-level algorithms to guide the AUVs towards the centre of an SGD. This facilitates the role of the PSO algorithm to locate the SGDs' centre. This also explains the decrease in the standard deviation of the accuracy in locating the global optimum achieved by the cooperative algorithms using the APP algorithm.

The next step in this research is to combine the cooperative search algorithms with inertia-levy flight [14]. The impact of the localisation, navigation and communication errors on the performance of this combination will be investigated. The results of the research will be tested and evaluated by applying them to a swarm of real AUVs cur-rently under development to locate an SGD location.

\section{References}

[1] Akawwi, E. J. Locating Zones and Quantify the Submarine Groundwater Discharge into the Eastern Shores of the Dead Sea-Jordan. PhD thesis, Göttingen Uni, Germany, 2006.

[2] Curey, R. K., Ash, M. E., Thielman, L. O., AND BArker, C. H. Proposed ieee inertial systems terminology standard and other inertial sensor standards. In PLANS 2004. Position Location and Navigation Symposium (IEEE Cat. No.04CH37556) (2004), pp. 83-90.

[3] El-Mihoub, T., Tholen, C., And Nolle, L. Informed search patterns for alleviating the impact of the localisation problem. In 33rd. European Conference on Modelling and Simulation ECMS 2019 (Caserta, Italy, 2019), pp. 1-6.

[4] Gordon, N. J., Salmond, D. J., And Smith, A. F. M. Novel approach to nonlinear/nongaussian bayesian state estimation. IEE Proceedings F - Radar and Signal Processing 140, 2 (1993), 107-113.

[5] Kalman, R. E. A New Approach to Linear Filtering and Prediction Problems. Journal of Basic Engineering 82, 1 (03 1960), 35-45.

[6] Leonard, J. J., And Durrant-Whyte, H. F. Mobile robot localization by tracking geometric beacons. IEEE Transactions on Robotics and Automation \%, 3 (1991), 376-382.

[7] Matousek, R., Popela, P., And Kudela, J. Heuristic approaches to stochastic quadratic assignment problem: Var and cvar cases. MENDEL 23, 1 (Jun. 2017), 73-78.

[8] Moore, W. S. The effect of submarine groundwater discharge on the ocean. Annual Review of Marine Science 2, 1 (2010), 59-88.

[9] Nelson, C. E., Donahue, M. J., Dulaiova, H., Goldberg, S. J., La Valle, F. F., 
Lubarsky, K., Miyano, J., Richardson, C., Silbiger, N. J., AND Thomas, F. I. Fluorescent dissolved organic matter as a multivariate biogeochemical tracer of submarine groundwater discharge in coral reef ecosystems. Marine Chemistry 177 (2015), $232-243$.

[10] Nolle, L. On a search strategy for collaborating autonomous underwater vehicles. Mendel (2015), 159-164.

[11] Prasser, D., And Dunbabin, M. Sensor network based auv localisation. In Field and Service Robotics (Berlin, Heidelberg, 2010), A. Howard, K. Iagnemma, and A. Kelly, Eds., Springer Berlin Heidelberg, pp. 285-294.

[12] Rigby, P., Pizarro, O., and Williams, S. B. Towards geo-referenced auv navigation through fusion of usbl and dvl measurements. In OCEANS 2006 (2006), pp. 1-6.

[13] Stutters, L., Liu, H., Tiltman, C., And Brown, D. J. Navigation technologies for autonomous underwater vehicles. IEEE Transactions on Systems, Man, and Cybernetics, Part $C$ (Applications and Reviews) 38, 4 (2008), 581-589.

[14] Tholen, C., El-Mihoub, T. A., Nolle, L., AND ZiElinski, O. On the robustness of self-adaptive levy-flight. In 2018 OCEANS MTS/IEEE Kobe Techno-Oceans (OTO) (2018), pp. $1-5$.

[15] Tholen, C., And Nolle, L. Parameter search for a small swarm of auvs using particle swarm optimisation. In Artificial Intelligence XXXIV (Cham, 2017), M. Bramer and M. Petridis, Eds., Springer International Publishing, pp. 384-396.

[16] Tholen, C., Nolle, L., And Werner, J. On the influence of localisation and communication error on the behaviour of a swarm of autonomous underwater vehicles. In Recent Advances in Soft Computing (Cham, 2019), R. Matoušek, Ed., Springer International Publishing, pp. 68-79.

[17] TVRDIK, J. Competition and cooperation in evolutionary algorithms: A comparative study. In MENDEL 2005, 11-th Int. Conference on Soft Computing (2005), pp. 108-113.

[18] Whitcomb, L., Yoerger, D., And Singh, H. Advances in doppler-based navigation of underwater robotic vehicles. In Proceedings 1999 IEEE International Conference on Robotics and Automation (Cat. No.99CH36288C) (1999), vol. 1, pp. 399-406.

[19] ZelinkA, I. SOMA - Self-Organizing Migrating Algorithm. Springer Berlin Heidelberg, Berlin, Heidelberg, 2004, pp. 167-217.

[20] Zielinski, O., Busch, J., Cembella, A., DAly, K., Engelbrektsson, J., HAnnides, A. K., AND SCHMIDT, H. Detecting marine hazardous substances and organisms: sensors for pollutants, toxins, and pathogens. Ocean Sci. 5 (September 2009), $329-349$. 